\title{
First report of Alternaria black spot of pomegranate caused by Alternaria alternata in Israel
}

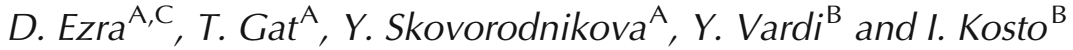 \\ A Department of Plant Pathology and Weed Research, ARO, The Volcani Center, Israel. \\ ${ }^{B}$ Ministry of Agriculture and Rural Development Extension Service, PO Box 6, Bet-Dagan 50250, Israel. \\ ${ }^{\mathrm{C}}$ Corresponding author. Email: dezra@volcani.agri.gov.il
}

\begin{abstract}
Black spot disease of pomegranate is a new disease caused by Alternaria alternata on pomegranates in Israel. Symptoms can be seen on the leaves and fruits but no damage to the inner edible tissue is found. The causal agent was isolated and Koch Postulates were demonstrated. This is the first report of black spot disease of pomegranate in Israel.
\end{abstract}

In 2004 a new disease was observed in pomegranate (Punica granatum) orchards in Israel. Black spot symptoms were noted on leaves and fruits, ranging from a single spot to more than $50 \%$ of the fruit surface (Fig. 1); some leaves became chlorotic and abscised (Fig. 2). Spots, which appeared at the start of the summer on all cultivars, were small $(1-3 \mathrm{~mm})$ and round on fruit and round to irregular $(1-4 \mathrm{~mm})$ on leaves. Each spot consisted of a green-yellow halo surrounding a necrotic lesion.

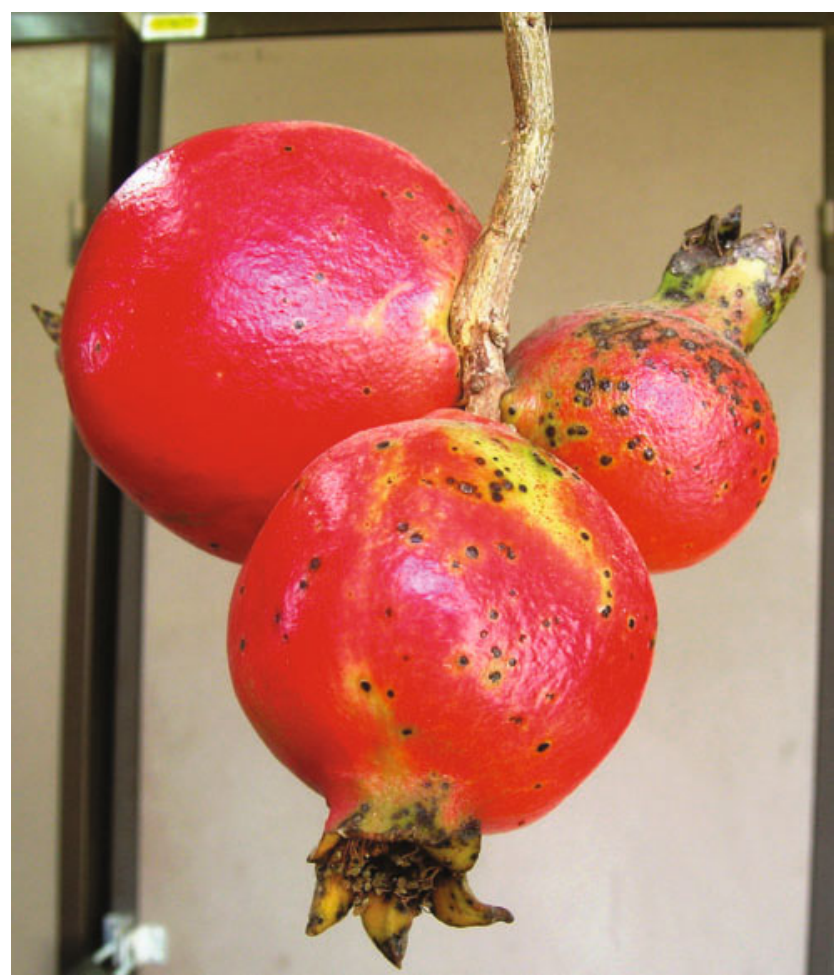

Fig. 1. Alternaria black spots of pomegranate fruits caused by A. alternata.
Damage to fruit was limited to the peel surface; the edible tissue was not damaged.

Symptomatic fruits and leaves were collected during the summer of 2007. Lesion pieces were plated on potato dextrose agar (PDA) amended with $12 \mu \mathrm{g} / \mathrm{L}$ tetracycline and incubated at $25^{\circ} \mathrm{C}$. Fungal colonies emerging from symptomatic tissue had morphology and conidia typical of Alternaria spp. Sequence analysis of the rDNA ITS region and $\beta$-tubulin gene of four different pathogenic isolates (A17, D2, 49, 53) from different cultivars were found to be identical (i.e. A17 GenBank accessions GQ240306, GQ240308), exhibiting 100\% identity to $A$. alternata (accessions FJ946480; EU139350). Polymerase chain reaction (PCR specific primers, apPCR-based) revealed differences between $A$. alternata isolated from pomegranates affected by 'black rot' and A. alternata isolated from 'black spot' disease

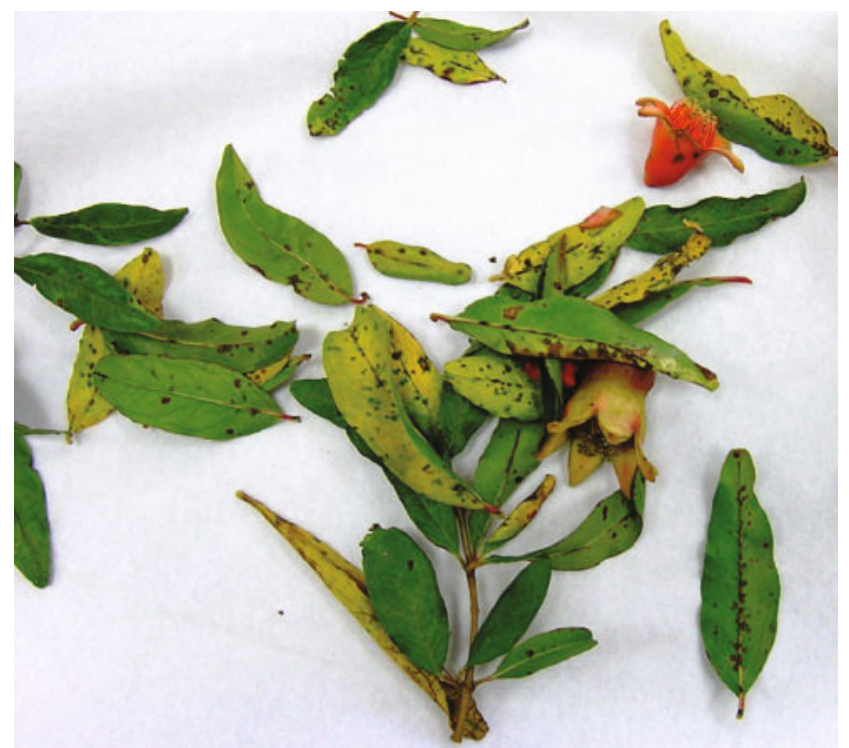

Fig. 2. Alternaria black spots of pomegranate leaves caused by A. alternata. 


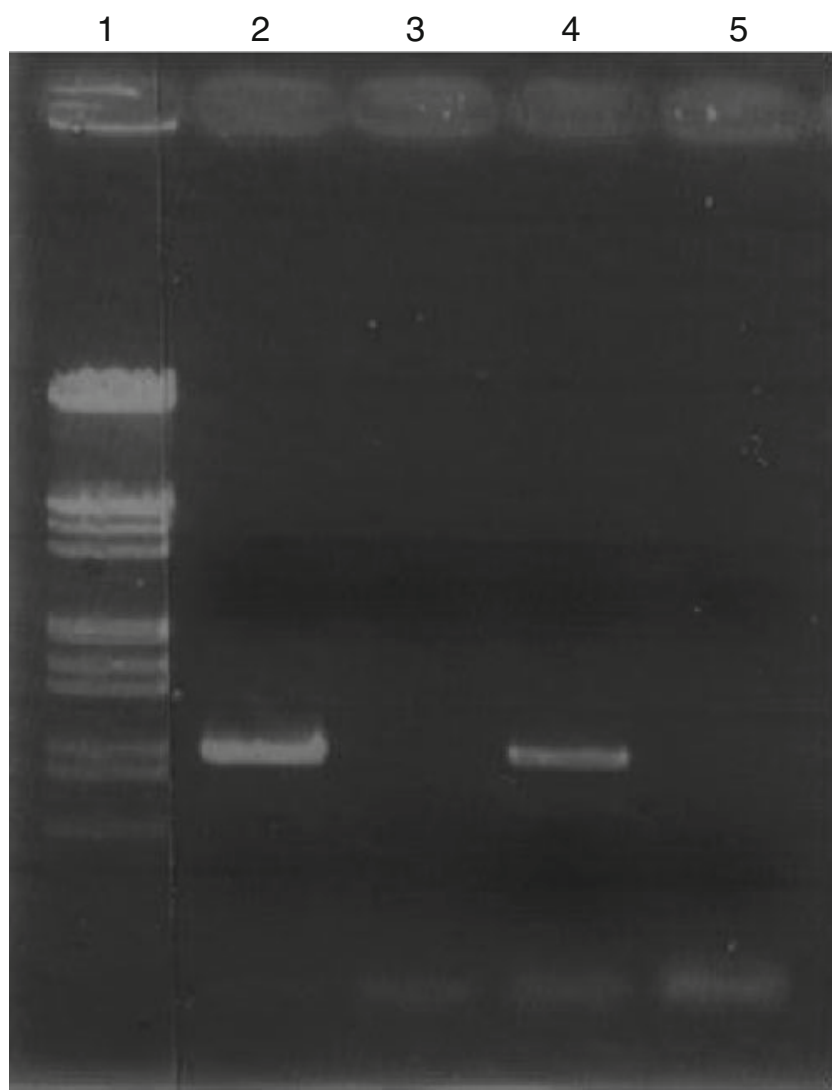

Fig. 3. Polymerase chain reaction (PCR) on A. alternata from pomegranate. Specific primers were designed based on differences found in apPCR performed on pathogenic and non-pathogenic isolates from pomegranate. 1. $\lambda$ HindIII/EcoRI DNA marker. 2. Isolate of A. alternata from black spot (displays symptoms on Pomegranate leaves and fruits). 3. A. alternata isolated from black rot infected Pomegranate (number 83). 4. Alternaria isolate from black spot. $5 . \mathrm{H}_{2} \mathrm{O}$ control.

(Fig. 3). Other Alternaria isolates from non-symptomatic pomegranate and other fruits, pathogenic and non-pathogenic, were not identified by the designed primers (data not shown).

Laboratory pathogenicity was assessed by detached leaf and fruit inoculation. Drops $(10 \mu \mathrm{L})$ of a $10^{6}$ conidia/mL suspension, from 50 different isolates, were inoculated on leaves ( 2 weeks

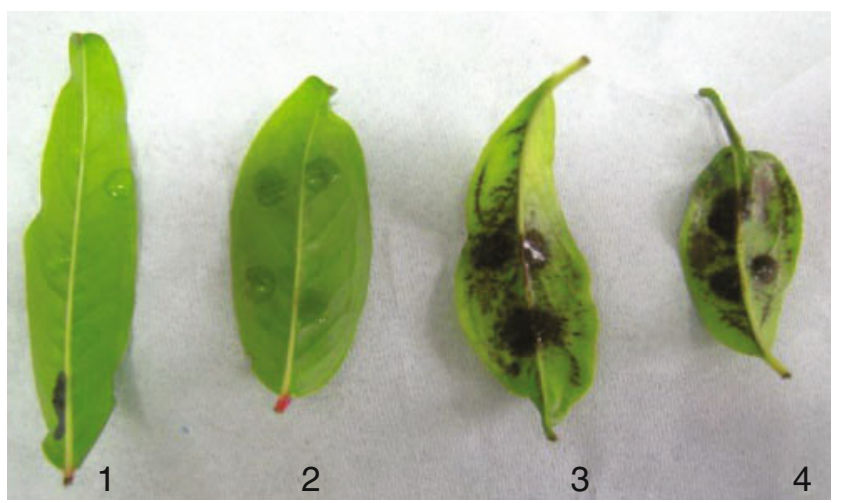

Fig. 4. Detached leaf inoculation test with pathogenic (black spot) and black $\operatorname{rot} A$. alternata isolates. The black rot isolate caused no symptoms while the pathogenic isolates caused necrosis of the inoculated area. $1 . \mathrm{H}_{2} \mathrm{O}$ control, 2. Black rot (internal rot) $A$. alternata isolate 83. 3. Pathogenic black spot A. alternata isolate 48. 4. Pathogenic black spot $A$. alternata isolate 43 .

old), fruit ( 2 months old) and flowers, placed in a moist chamber $\left(25^{\circ} \mathrm{C}\right)$. Symptoms appeared $48-72 \mathrm{~h}$ later and were similar to those observed in the field. Koch's Postulates were completed by inoculation of greenhouse plants $\left(25^{\circ} \mathrm{C}, 14 \mathrm{~h}\right.$ photoperiod). Plants (3-6 months old), cvv. Wonderful and Shami, were sprayed or drop-inoculated with a $10^{6}$ conidia/mL suspension and covered with plastic bags for 1 month. Black spots appeared on leaves approximately 1 week after inoculation; the fungus was reisolated from infected leaves. Alternaria alternata from 'black rot' did not cause symptoms when inoculated on leaves (Fig. 4). This is the first report of A. alternata causing black spot disease of pomegranate in Israel. We believe that this is the first record of Alternaria black spot on pomegranate fruit while leaf spot has been previously reported in India (Madhukar and Reddy 1988).

\section{Acknowledgements}

This research was financed by the Israeli Pomegranate Marketing Council.

\section{Reference}

Madhukar J, Reddy SM (1988) Some new leaf spot diseases of pomegranate. Indian Journal of Mycology and Plant Pathology 18, 171-172.

Manuscript received 24 November 2009, accepted 3 December 2009 